\title{
UBR5 oncogene as an indicator of poor prognosis in gastric cancer
}

\author{
FANGHUI DING $^{1,2}$, XIAOLIANG ZHU ${ }^{1,2}$, XIAOJING SONG $^{1,2}$, PEI YUAN $^{1}$, \\ LONGFEI REN $^{1,2}$, CHANGPENG CHAI ${ }^{1,3}$, WENCE ZHOU ${ }^{1,3}$ and XUN LI ${ }^{1,2}$ \\ ${ }^{1}$ The First Clinical Medical College of Lanzhou University; ${ }^{2}$ General Surgery Department Ward V; \\ ${ }^{3}$ General Surgery Department Ward II, Lanzhou University First Hospital, Lanzhou, Gansu 730000, P.R. China
}

Received January 1, 2020; Accepted July 21, 2020

DOI: $10.3892 /$ etm.2020.9135

\begin{abstract}
The human ubiquitin protein ligase E3 component $\mathrm{N}$-recognin 5 (UBR5) gene, which is localized to chromosome $8 \mathrm{q} 22$, encodes an $\sim 10 \mathrm{~kb}$ mRNA and a $>300 \mathrm{kDa}$ protein, which can be detected in a number of cell types. UBR5 is implicated in several types of cancer, including ovarian cancer, gallbladder cancer and lymphoma; however, its role in gastric cancer is not completely understood. In the present study, the expression levels of UBR5 in human gastric cancer tissues and cell lines were examined via immunohistochemistry, reverse transcription-quantitative PCR analysis and western blotting. Furthermore, the association between UBR5 expression and clinicopathological characteristics, as well as the prognosis of patients with gastric cancer, were examined. In addition, the role of UBR5 in gastric cancer cell proliferation, invasion and migration was investigated by conducting MTS, Transwell and wound healing assays, respectively. The results indicated that the mRNA and protein expression levels of UBR5 were significantly increased in gastric cancer tissues compared with para-carcinoma tissues. High UBR5 expression levels were significantly associated with larger tumor size, advanced TNM stage and lymph node metastasis. In addition, high UBR5 expression indicated a poor prognosis in patients with gastric cancer. Furthermore, in vitro experiments demonstrated that UBR5 knockdown was associated with reduced HGC-27 gastric cancer cell proliferation, invasion and migration compared with the small interfering RNA control group. Therefore, the results indicated that UBR5 may serve a key role in gastric cancer, indicating that UBR5 may also serve as an important prognostic biomarker.
\end{abstract}

Correspondence to: Dr Xun Li, The First Clinical Medical College of Lanzhou University, 1 Dong Gang Xi Lu, Chengguan, Lanzhou, Gansu 730000, P.R. China

E-mail: 1xdr21@126.com

Key words: ubiquitin protein ligase E3 component N-Recognin 5, gastric cancer, proliferation, migration, invasion

\section{Introduction}

Gastric cancer is the second most common malignancy and cause of cancer-related mortality in China, ranking with the highest incidence and mortality among all malignancies in Northwest China $(1,2)$. Although surgery is the primary therapeutic strategy for gastric cancer, studies have suggested that an enhanced understanding of the molecular mechanisms underlying gastric tumorigenesis may enable the identification of novel diagnostic and prognostic markers, as well as the development of novel molecular drugs $(3,4)$. The molecular pathogenesis underlying gastric cancer is not completely understood and requires further investigation, which is particularly important for patients with gastric cancer in high prevalence areas.

The human ubiquitin protein ligase E3 component $\mathrm{N}$-recognin 5 (UBR5) gene, which is localized to chromosome $8 q 22$, encodes an $\sim 10 \mathrm{~kb}$ mRNA and a $>300 \mathrm{kDa}$ protein, which is abundantly expressed in various types of cells (5-7). The UBR5 protein belongs to the E6-AP carboxyl terminus family, which serve as E3 ubiquitin-protein ligases and target specific proteins for ubiquitin-induced proteolysis (5-7). UBR5 is an important nuclear phosphate protein, which is involved in DNA damage response, cell proliferation, cell cycle arrest, metabolism and apoptosis (8-13). Previous studies have implicated UBR5 in several types of cancer, including ovarian cancer, gallbladder cancer and lymphoma $(5-7,14,15)$. Originally, UBR5 was identified in a screen for progestin-regulated genes in breast cancer cells as a novel isolate progestin-regulated gene (16). UBR5 was also reported to serve an important role in the pathogenesis, development and therapeutic resistance of other progestin-related types of cancer, such as ovarian cancer (17-19). The functions of UBR5 have been reported in several types of cancer, indicating the role of UBR5 in the pathogenesis of cancer (5). However, although disrupted UBR5 expression has been reported in gastric cancer (20), the role of UBR5 in gastric cancer cell proliferation, migration and invasion is not completely understood.

In the present study, UBR5 mRNA and protein expression levels were investigated in patients with gastric cancer, and the association between survival time and UBR5 expression was examined. The aim of the present study was to improve the current understanding of the role of UBR5 in the biological functions of gastric cancer cells, and UBR5 knockdown was employed to explore the effects of UBR5 in vitro. 


\section{Materials and methods}

Patients and cancer samples. The present study was approved by the Institutional Review Board of Lanzhou University First Hospital (approval no. LDYYLL2020-235). All procedures were conducted in accordance with the principles outlined in the Declaration of Helsinki, and exception to the requirement of informed consent was approved by the Institutional Review Board of Lanzhou University First Hospital. Gastric cancer and para-carcinoma tissue (5 $\mathrm{cm}$ from the tumor margin) samples were collected at the Lanzhou University First Hospital between January 2012 and December 2012. A section of the samples were sent to the Department of Pathology for clinicopathological examination and immunohistochemical staining. The remainder of the samples were transferred to the lab and stored in liquid nitrogen until further analysis. The patients included in the present study were initially diagnosed with gastric cancer, and none had received chemotherapy or radiotherapy prior to surgery. The clinicopathological information of the patients was also collected for analysis and is presented in Table I.

Cell culture and small interfering (si)RNA knockdown of UBR5. The gastric cell line HGC-27 $(21,22)$ and the gastric mucosal epithelial cell line GES-1 $(23,24)$ were purchased from China Infrastructure of Cell Line Resources, Institute of Basic Medical Sciences, Chinese Academy of Medical Sciences. Cells were cultured in RPMI-1640 (HyClone; Cytiva) supplemented with $10 \%$ FBS (HyClone; Cytiva) and $1 \%$ penicillin-streptomycin (Beijing Solarbio Science \& Technology Co.,Ltd.). UBR5 siRNA and negative control (NC) siRNA were purchased from Wuhan GeneCreate Biological Engineering Co., Ltd. The sequences of the UBR5 siRNAs transfected were as follows: 1, forward 5'-CGGGAAAGG GAGAGAGAAATT-3' and reverse, 5'-UUUCUCUCUCCC UUUCCCGTT-3'; 2 forward, 5'-GGUCAAUAGUAGAGA AGAUTT-3' and reverse, 5'-AUCUUCUCUACUAUUGAC CTT-3'; 3 forward, 5'-GAAAUAUCCUCAAGUGAAATT-3' and reverse, 5'-UUUCACUUGAGGAUAUUUCTT-3'. The sequences of the NC siRNA wereas follows: Forwad, 5'-UUC UCCGAACGUGUCACGUTT-3' and reverse, 5'-ACGUGA CACGUUCGGAGAATT-3'. Cells were seeded $\left(1 \times 10^{6} / \mathrm{ml}\right)$ into 6-well (for the migration assay) or 96-well plates (for the cell proliferation assay). Following culture for $24 \mathrm{~h}$, when cell confluence reached $70-80 \%$, cells were transfected with $50 \mathrm{~nm}$ UBR5 siRNA or $50 \mathrm{~nm}$ NC siRNA using Lipofectamine ${ }^{\circledR}$ 3000 (Invitrogen; Thermo Fisher Scientific, Inc.). At 48-72 h post-transfection, cells were used for subsequent experiments.

Reverse transcription-quantitative PCR (RT- $q P C R)$. RT-qPCR analysis $\left(2^{-\Delta \Delta \mathrm{Cq}}\right.$ method) was performed to detect the mRNA expression level of UBR5 (25). Total RNA was extracted using TRIzol ${ }^{\circledR}$ reagent (Invitrogen; Thermo Fisher Scientific, Inc.). The concentration and purity of the extracted RNA was determined using a NanoDrop 2000 Spectrophotometer (Thermo Fisher Scientific, Inc.). Subsequently, total RNA was reverse transcribed into cDNA using the high-capacity cDNA Reverse Transcriptase kit (Invitrogen; Thermo Fisher Scientific, Inc.) with the following temperature protocol: $25^{\circ} \mathrm{C}$ for $10 \mathrm{~min}$, $37^{\circ} \mathrm{C}$ for $120 \mathrm{~min}$ and $85^{\circ} \mathrm{C}$ for $5 \mathrm{~min}$. Subsequently, qPCR was performed in a final volume of $20 \mu \mathrm{l}$ consisting of cDNA, qPCR mix (Beijing Transgen Biotech Co., Ltd.), primers and water, with the Real-time PCR system (Applied Biosystems; Thermo Fisher Scientific, Inc.). The following thermocycling conditions were used for qPCR: $94^{\circ} \mathrm{C}$ for $30 \mathrm{sec} ; 40$ cycles of $94^{\circ} \mathrm{C}$ for $5 \mathrm{sec}$ and $60^{\circ} \mathrm{C}$ for $30 \mathrm{sec}$. The following primers were used for qPCR: UBR5 forward, 5'-TGATCCTGAGCC TTTGCCAG-3' and reverse, 5'-GCGCTTTCGGTTTTCCTG TA-3'; and GAPDH forward, 5'-AATGGGCAGCCGTTAGGA AA-3' and reverse, 5'-GCGCCCAATACGACCAAATC-3'. The receiver operating characteristics curve analysis was applied to identify the optimal cut off for grouping patients by UBR6 expression. The area under the curve was 0.7022 and the cut off was 0.9945 . Therefore, expression $<0.9945$ was defined as low level, whereas $>0.9945$ was defined as high level.

Western blotting. Total protein was extracted from clinical samples and cells using RIPA buffer (Sigma-Aldrich; Merck KGaA) with protease inhibitor cocktail (Roche Diagnostics). Protein concentration was determined by using a BicinChoninic Acid kit (Pierce; Thermo Fisher Scientific, Inc.). Equal amounts of proteins (human tissue, $10 \mu \mathrm{g} / \mathrm{lane}$; cell line, $30 \mu \mathrm{g} / \mathrm{lane}$ ) were separated via 10\% SDS-PAGE and transferred to a nitrocellulose membrane (Amersham; Cytiva), followed by blocking with 5\% not-fat milk powder in PBS for $1 \mathrm{~h}$ at room temperature. The membranes were incubated overnight at $4^{\circ} \mathrm{C}$ with primary antibodies targeted against: UBR5 (cat. No. ab70311; 1:1,000; Abcam) and $\beta$-actin (cat. No. sc-47778; 1:1,000; Santa Cruz Biotechnology, Inc.). Following washing three times with PBST $(0.1 \%$ Tween-20) for $5 \mathrm{~min}$, the membranes were incubated with horseradish peroxidase-labeled goat anti-mouse and goat anti-rabbit secondary antibodies (cat. Nos. sc-2005 and sc-2004; Santa Cruz Biotechnology, Inc.) for $2 \mathrm{~h}$ at room temperature. Protein bands were visualized using a chemiluminescence kit (Merck $\mathrm{KGaA}$ ). Protein analysis was performed by using Image $\mathbf{J}$ (version 2.1.4; National Institutes of Health).

Immunohistochemistry. Prior to embedding with paraffin, gastric cancer and para-carcinoma tissues were fixed using $10 \%$ paraformaldehyde for $48-72 \mathrm{~h}$ at room temperature. Then they were cut into $4-\mu \mathrm{m}$ sections. The sections were deparaffinized in xylene and rehydrated in descending alcohol series. After rehydration, sections were washed with PBS at room temperature. Then, following antigen retrieval at $92^{\circ} \mathrm{C}$ for $2 \mathrm{~h}$ using Citrate Antigen Retrieval Solution (Beijing Leagene Biotechnology Co., Ltd.) and blocking with 5\% BSA (Beijing Leagene Biotechnology Co., Ltd.) at $4^{\circ} \mathrm{C}$ for $2 \mathrm{~h}$, the sections were incubated overnight at $4^{\circ} \mathrm{C}$ with an anti-UBR5 primary antibody (cat. No. ab70311; 1:500; Abcam). Subsequently, the sections were rinsed three times with PBS and incubated with a goat anti rabbit secondary antibody for $1 \mathrm{~h}$ at room temperature (cat. no. SP 2001; 1:200; OriGene Technologies, Inc.). Chromogen detection was performed using a DAB kit (OriGene Technologies, Inc.). Images were obtained using DP72 digital light microscope (Olympus Corporation; magnification, $\mathrm{x} 200$ ).

Cell proliferation assay. Cells $\left(1 \times 10^{6} / \mathrm{ml}\right)$ were cultured in 96-well plates with RPMI-1640/10\% FBS medium. After 
Table I. Clinicopathological characteristics and UBR5 expression in patients with gastric cancer.

\begin{tabular}{|c|c|c|c|c|}
\hline \multirow[b]{2}{*}{ Variable } & \multirow[b]{2}{*}{ Total (n) } & \multicolumn{2}{|c|}{$\begin{array}{l}\text { UBR5 } \\
\text { expression } \\
\text { (n) }\end{array}$} & \multirow[b]{2}{*}{ P-value } \\
\hline & & Low & High & \\
\hline Gender & & & & 0.7009 \\
\hline Male & 75 & 25 & 50 & \\
\hline Female & 37 & 11 & 26 & \\
\hline Age (years) & & & & 0.5826 \\
\hline$\geq 56$ & 54 & 16 & 38 & \\
\hline$<56$ & 58 & 20 & 38 & \\
\hline Tumor size $(\mathrm{cm})$ & & & & $<0.05$ \\
\hline$\geq 3$ & 97 & 25 & 72 & \\
\hline$<3$ & 15 & 11 & 4 & \\
\hline TNM stage & & & & $<0.05$ \\
\hline I & 19 & 10 & 9 & \\
\hline II, III & 93 & 26 & 77 & \\
\hline Lymph node metastasis & & & & $<0.05$ \\
\hline Positive & 79 & 19 & 60 & \\
\hline Negative & 33 & 17 & 16 & \\
\hline UBR5 expression & & & & \\
\hline$\geq 0.9945$ & 76 & & & \\
\hline$<0.9945$ & 36 & & & \\
\hline
\end{tabular}

cell confluence reached 70-80\%, transfection was performed. Following transfection for $48 \mathrm{~h}$, the MTS assay (Promega Corporation) was performed according to the manufacturer's protocol. Briefly, following incubation with MTS at $37^{\circ} \mathrm{C}$ for $4 \mathrm{~h}$, the absorbance of each well was measured at a wavelength of $490 \mathrm{~nm}$ using a microplate reader (BioTek Instruments, Inc.).

Cell invasion and migration assays. Cell invasion was assessed using 6-well Transwell chambers (Corning, Inc.). Cells were seeded into the upper chambers $\left(5 \times 10^{4}\right.$ cells $\left./ \mathrm{ml}\right)$ with serum-free RPMI-1640 medium. The lower chambers were pre-coated with Matrigel $(50 \mathrm{mg} / \mathrm{l})$ for $37^{\circ} \mathrm{C}$ for $1 \mathrm{~h}$. RPMI-1640 medium containing 10\% FBS was subsequently added to the lower chamber and then incubated at $37^{\circ} \mathrm{C}$ for $24 \mathrm{~h}$. Invading cells were stained with hematoxylin for $5 \mathrm{~min}$ at room temperature. Invading cells were visualized using a light microscope (Olympus Corporation; magnification, $\mathrm{x} 200$ ) in five randomly selected fields of view. Cell migration was assessed using a wound healing assay. At $48 \mathrm{~h}$ post-transfection, the cell layer was scratched with a sterile $200-\mu 1$ pipette tip. After washing with PBS, cells were cultured with serum-free RPMI- 1640 for $24 \mathrm{~h}$ at $37^{\circ} \mathrm{C}$. Images of the wound were captured at 0 and $24 \mathrm{~h}$ using a DP72 digital light micropscope (Olympus Corporation; magnification, x200). Cell migration was quantified by calculating the percentage of migration area covered with Image J (v2.1.4; National Institutes of Health).
Statistical analysis. Data are presented as the mean \pm standard deviation and at least three independent experiments were performed per experiment. Comparisons between two groups were analyzed using an unpaired Student's t-test. Comparisons among multiple groups were analyzed using one-way ANOVA followed by Tukey's post hoc test. The predicted significance of UBR5 was assessed using Kaplan-Meier analysis and the log-rank test. The association between UBR5 expression and clinicopathological variables was examined using the $\chi^{2}$ test. Statistical analyses were conducted using GraphPad Prism software (v5; GraphPad Software, Inc.).

\section{Results}

Characteristics of patients with gastric cancer. As shown in Table I, 112 patients (75 male patients and 37 female patients; median age, 56 years; age range, 24-80 years) with gastric cancer were included in the present study. The size of the tumor was $\geq 3 \mathrm{~cm}$ in 97 patients and $<3 \mathrm{~cm}$ in 15 patients. A total of 19 patients had TNM (26) stage I, whereas 93 patients had advanced TNM stage (II and III). Lymph node metastasis was detected in 79 patients, whereas 33 patients were negative for lymph node metastasis.

Expression of UBR5 in human gastric cancer. The mRNA and protein expression levels of UBR5 were examined in gastric cancer and para-carcinoma tissue samples. The mRNA expression levels of UBR5 were significantly increased in gastric cancer tissue samples compared with para-carcinoma tissue samples (Fig. 1A). Regarding the mRNA expression of UBR5, 76 cases were in the high expression group $(>0.9945$ fold) and 36 cases were in the low expression group $(<0.9945$ fold). A total of 43 gastric cancer samples and 20 para-carcinoma samples were examined via western blotting. The protein expression level of UBR5 was significantly increased in gastric cancer tissues compared with para-carcinoma tissue samples (Fig. 1B). The protein expression of UBR5 was also detected via immunohistochemistry. The results indicated that the expression of UBR5 was increased in gastric cancer samples compared with para-carcinoma samples (Fig. 1D).

Association between UBR5 expression, clinicopathological characteristics and prognosis of patients with gastric cancer. Increased expression of UBR5 in gastric cancer tissues suggested that UBR5 may serve an important role in the development of gastric cancer. High UBR5 expression was significantly associated with larger tumor size, advanced TNM stage and lymph node metastasis $(\mathrm{P}<0.05$; Table I). To further explore the role of UBR5 in the prediction of patient survival, the association between the mRNA expression level of UBR5 and survival was analyzed. The results indicated that higher UBR5 expression levels were associated with poorer prognosis in patients with gastric cancer compared with lower UBR5 expression levels ( $\mathrm{P}<0.001$; Fig. 2).

mRNA and protein expression levels of UBR5 in cancer and control cell lines. To understand the role of UBR5 in the pathogenesis of gastric cancer, the HGC-27 gastric cell line and the GES-1 gastric mucosal epithelial cell line were used in present study. UBR5 mRNA and protein expression levels were 

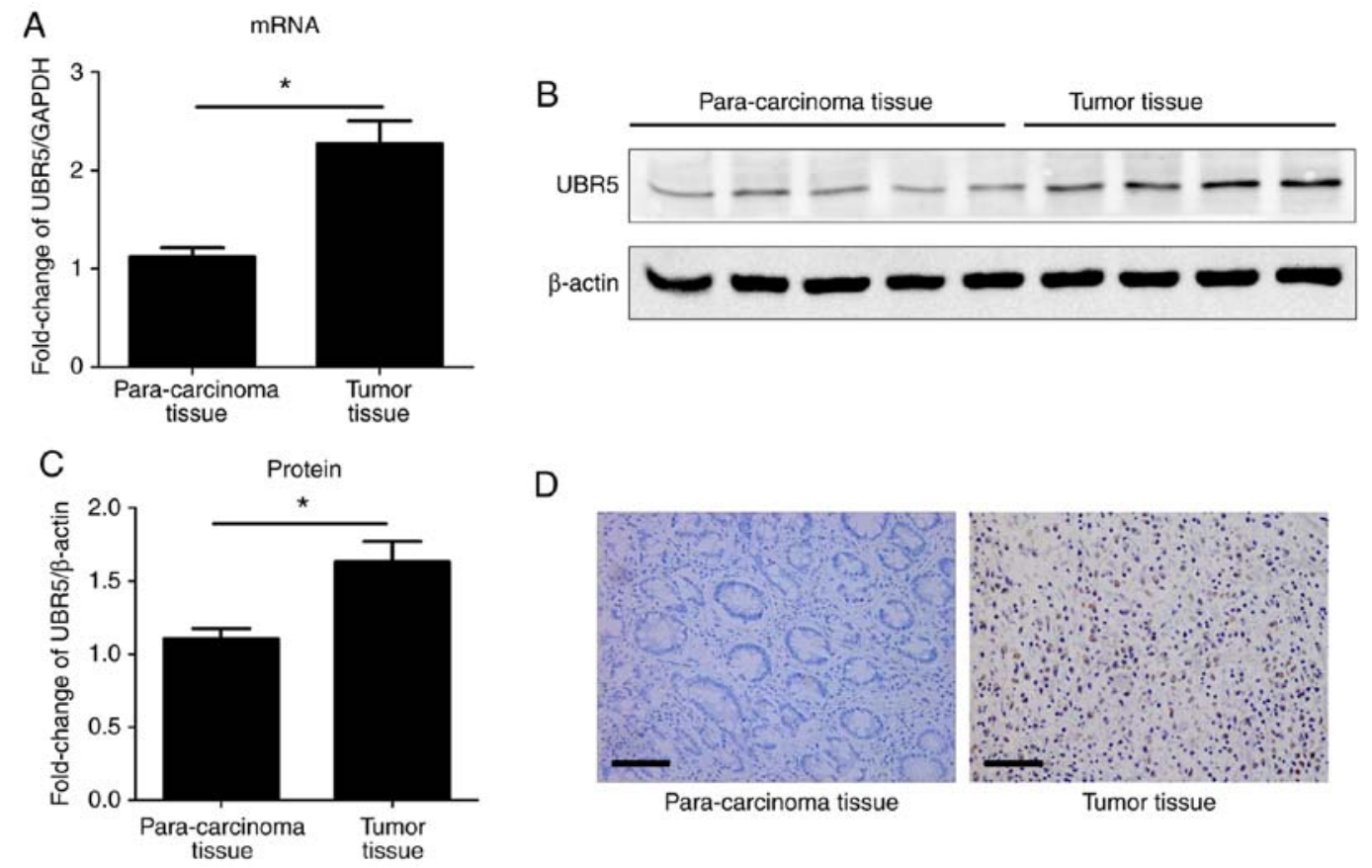

Figure 1. UBR5 mRNA and protein expression levels are increased in patients with gastric cancer. (A) UBR5 mRNA expression levels were increased in gastric cancer tissues compared with para-carcinoma tissues. UBR5 protein expression levels were (B) determined by western blotting and (C) semi-quantified. (D) UBR5 protein expression was examined via immunohistochemistry (magnification, $\mathrm{x} 200$; scale bar, $50 \mu \mathrm{m}$ ). ${ }^{*} \mathrm{P}<0.05$. UBR5, ubiquitin protein ligase E3 component N-recognin 5.

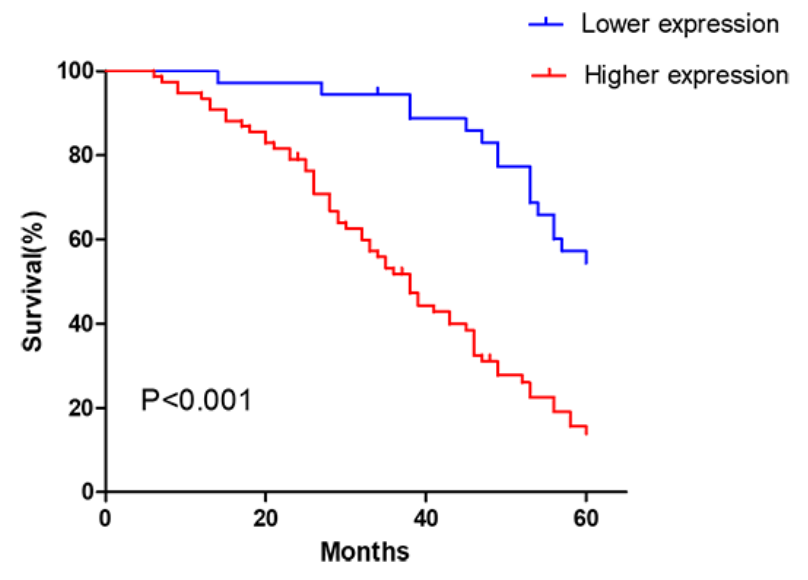

Figure 2. Kaplan-Meier survival analysis of UBR5 expression in patients with gastric cancer. High UBR5 expression ( $\geq 0.9945$ fold mRNA level) was associated with shorter survival time, whereas low UBR5 expression $(<0.9945$ fold mRNA level) was associated with longer survival time. UBR5, ubiquitin protein ligase $\mathrm{E} 3$ component $\mathrm{N}$-recognin 5 .

examined in vitro. The mRNA and protein expression levels of UBR5 were significantly increased in gastric cancer cells compared with control gastric mucosal epithelial cells (Fig. 3).

Effect of UBR5 knockdown on gastric cancer cell proliferation, invasion and migration. According to the aforementioned results, increased expression of UBR5 indicated that inhibition of UBR5 might be beneficial for the treatment of gastric cancer. Therefore, UBR5 knockdown was performed using three UBR5-targeting siRNAs in the present study. All three siRNAs significantly decreased UBR5 expression levels compared with the control siRNA group; however, the lowest level of UBR5 expression was observed in the 3\# siRNA group. Thus, UBR5-targeting 3\# siRNA was used in subsequent experiments. Strong proliferation, invasion and migration abilities are the classical biological properties of cancer cells (27). To examine whether UBR5 knockdown effectively inhibited malignant biological properties, MTS, Transwell and wound healing assays were performed. A higher optical density value at $490 \mathrm{~nm}$ was observed in the UBR5 siRNA group compared with the control siRNA group, which indicated that UBR5 knockdown decreased cell proliferation. Additionally, a reduced number of invading cells and fewer changes in the gap distance were observed in the UBR6 siRNA group compared with the control siRNA group, suggesting that UBR5 knockdown decreased cancer cell invasion and migration (Fig. 4D-G).

\section{Discussion}

UBR5 serves as an important regulator in several types of cancer, including ovarian cancer, gallbladder cancer and lymphoma $(5-7,14,15)$. The present study investigated the role of UBR5 in the pathogenesis and development of gastric cancer. The results indicated that UBR5 $\mathrm{mRNA}$ and protein expression levels were significantly increased in gastric cancer samples compared with para-carcinoma samples. Similarly, UBR5 expression levels were upregulated in the HGC-27 gastric cancer cell line compared with control mucosal epithelial cells. The dysregulated expression indicated that UBR5 may be involved in the pathogenesis of gastric cancer.

To further elucidate the role of UBR5 in gastric cancer, the association between the expression of UBR5 and clinicopathological characteristics in gastric cancer samples was investigated in the present study. Higher UBR5 expression was associated 

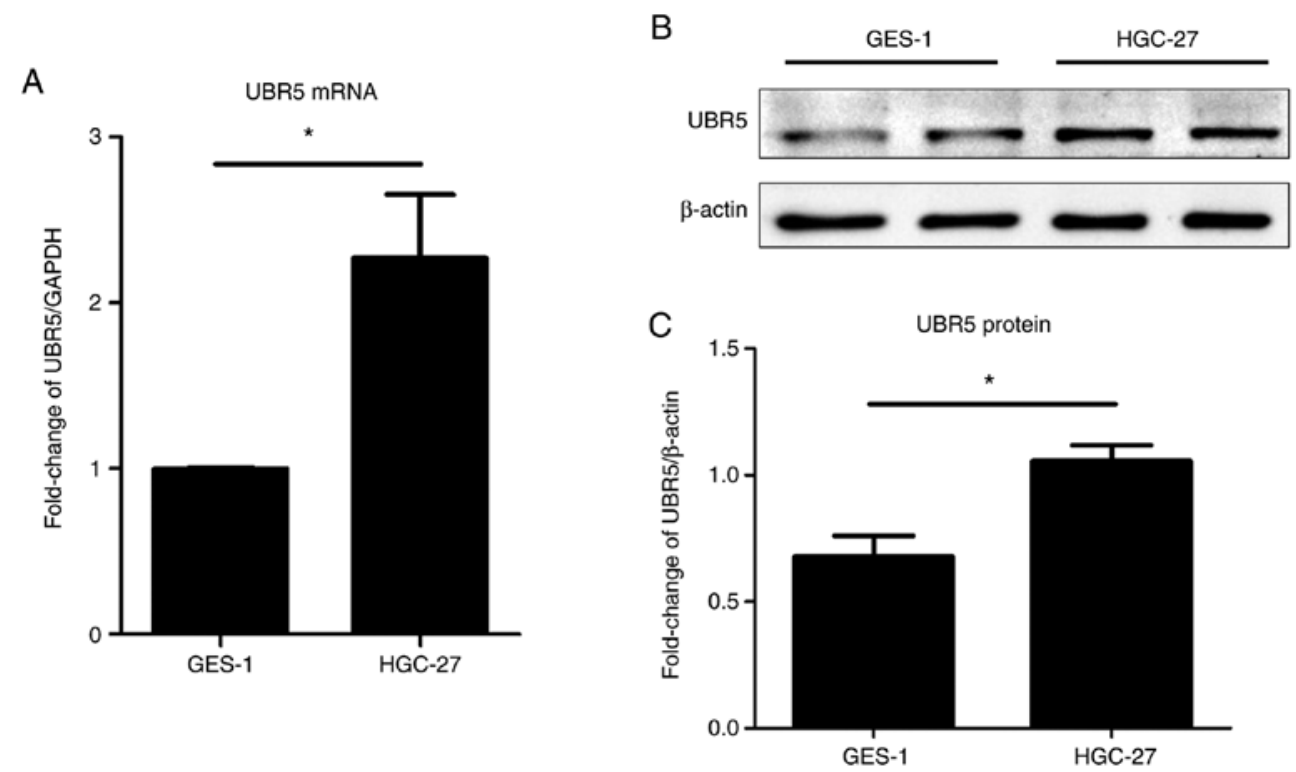

Figure 3. UBR5 mRNA and protein expression levels are increased in the HGC-27 gastric cancer cell line. (A) UBR5 mRNA expression levels were increased in HGC-27 cells compared with GES-1 control cells. UBR5 protein expression levels were (B) determined by western blotting and (C) semi-quantified. ${ }^{*} \mathrm{P}<0.05$. UBR5, ubiquitin protein ligase $\mathrm{E} 3$ component $\mathrm{N}$-recognin 5.
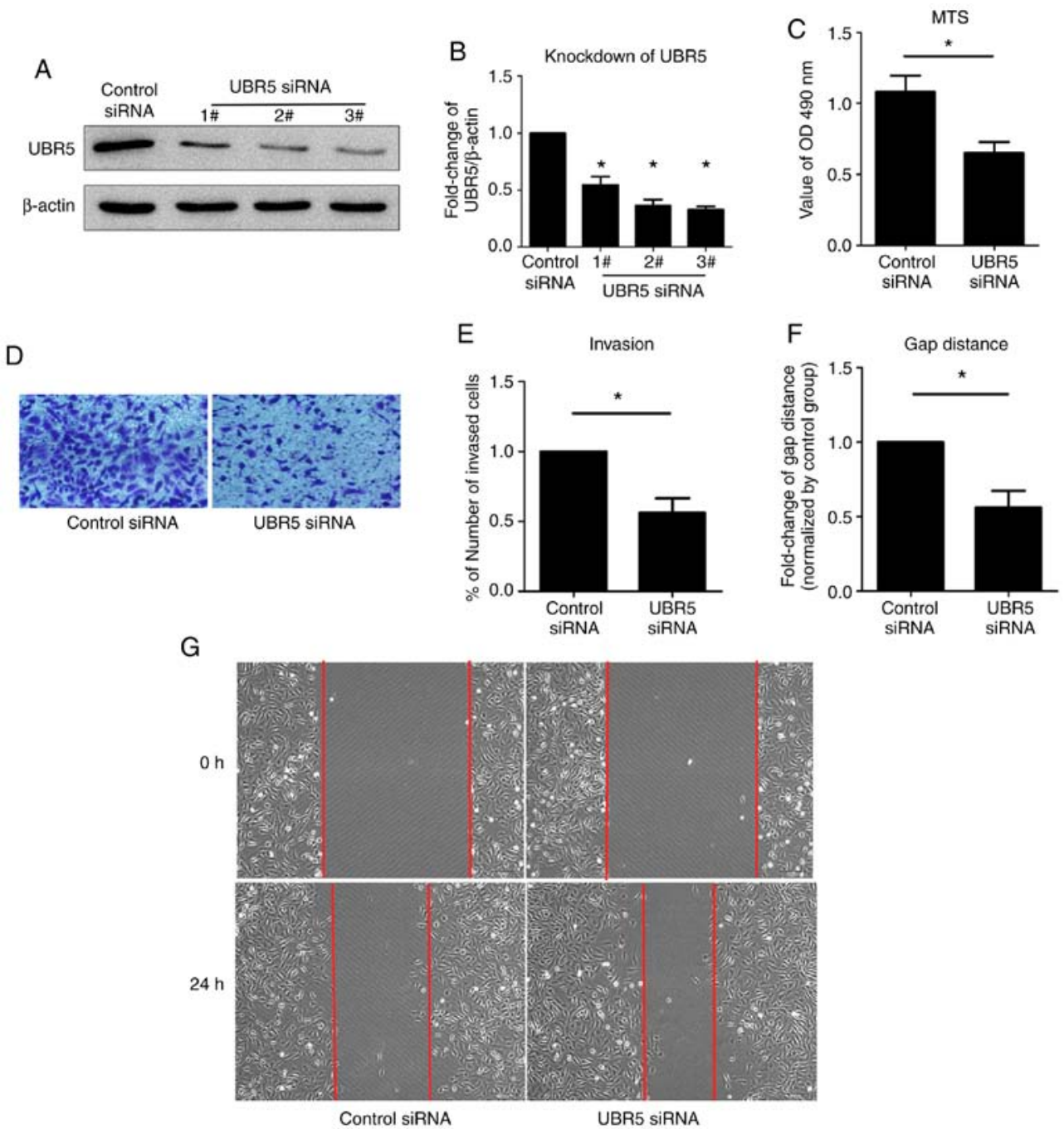

Figure 4. UBR5 knockdown inhibits gastric cancer cell proliferation, invasion and migration. The transfection efficiency of UBR5 siRNAs was (A) determined by western blotting and (B) semi-quantified. (C) Cell proliferation was assessed by performing an MTS assay. Cell invasion was (D) assessed using a Transwell invasion assay (magnification, $\mathrm{x} 200$ ) and (E) quantified. (F) Cell migration was assessed by performing a wound healing assay. (G) Representative images of the wound healing assay (magnification, $\mathrm{x} 200$ ). ${ }^{*} \mathrm{P}<0.05$ vs. control siRNA. UBR5, ubiquitin protein ligase E3 component $\mathrm{N}$-recognin 5; siRNA, small interfering RNA. 
with larger tumor size, advanced TNM stages and lymph node metastasis, suggesting that increased expression of UBR5 indicated poorer prognosis. Moreover, the association between the expression of UBR5 and survival was investigated. The higher the expression of UBR5 in cancer tissues, the shorter the survival time of patients with gastric cancer. Previous studies indicated a similar role of UBR5 in other types of cancer, such as ovarian cancer and gallbladder cancer $(5-7,14)$. The results indicated that increased UBR5 expression levels may serve an important role in the development and progression of gastric cancer. Therefore, UBR5 may serve as an oncogenic factor and may be considered as a biomarker or prognosis predictor in gastric cancer.

Based on the finding that increased UBR5 expression favored tumor development, UBR5 inhibition may serve as a potential therapeutic strategy for gastric cancer. In addition, the HGC-27 gastric cancer cell line was used in the present study. After selecting the most effective siRNA, basic tumor properties were examined in vitro. Compared with the control siRNA group, UBR5 knockdown inhibited cancer cell proliferation, invasion and migration. However, a minor limitation of the migration assay was that it could not be guaranteed that the images obtained at 0 and $24 \mathrm{~h}$ were from the same field of view. Despite the limitation, the results further supported the use of UBR5 inhibition as a therapeutic strategy for gastric cancer.

UBR5 serves an important role in DNA damage response, metabolism, transcription and apoptosis (8-13). In the present study, the in vitro results demonstrated that UBR5 may also serve a role in cell proliferation, invasion and migration. UBR5 is a member of the E3 ubiquitin ligases, which are key regulators in the ubiquitin-proteasome system $(6,7)$. In addition, UBR5 interacts with several proteins and signaling pathways involved in a wide variety of cellular processes, including the cell cycle, transcriptional and translational machinery $(9,14,28,29)$. The PI3K/Akt signaling pathway is associated with cell survival and proliferation in various types of cancer $(14,30)$, indicating that the role of UBR5 in gastric cancer proliferation may be mediated via the PI3K/Akt signaling pathway. In addition, UBR5, acting as an ubiquitin ligase, can directly degrade modulator of apoptosis protein 1 (MOAP-1) by ubiquitylation, thereby inhibiting MOAP-1 stability, and MOAP-1 exerts an effect by enhancing the expression of the proapoptotic protein-Bax $(9,19)$. Therefore, whether inhibition of UBR5 can decrease gastric cancer cell proliferation via MOAP-1 requires further investigation. Future studies should focus on the molecular mechanism underlying the role of UBR5 in the development and progression of gastric cancer.

In summary, the present study demonstrated that increased expression levels of UBR5 were associated with poor prognosis in patients with gastric cancer, whereas UBR5 knockdown decreased gastric cancer cell proliferation, invasion and migration.

\section{Acknowledgements}

Not applicable.

\section{Funding}

The present study was supported by the Health Industry Research Project of Gansu Province (grant no. GSWSKY2017-26), the
Gansu Province Science Foundation for Distinguished Young Scholars (grant no. 1606RJDA317), the Key Laboratory of Biotherapy and Regenerative Medicine of Gansu Province (grant no. zdsyskfkt- 201704) and the Foundation of The First Hospital of Lanzhou University (grant no. ldyyyn2015-16).

\section{Availability of data and materials}

The datasets used and/or analyzed during the present study are available from the corresponding author on reasonable request.

\section{Authors' contributions}

FD, WZ and XL designed the study. FD, XZ, XS and LR collected the samples, analyzed the data and wrote the manuscript. FD, PY and CC conducted the literature search and collected the data. FD, PY and XS performed the experiments and collected the data. All authors read and approved the final manuscript.

\section{Ethics approval and consent to participate}

The present study was approved by the Institutional Review Board of Lanzhou University First Hospital (approval no. LDYYLL2020-235). All procedures were conducted in accordance with the principles outlined in the Declaration of Helsinki, and exception to the requirement of informed consent was approved by the Institutional Review Board of Lanzhou University First Hospital.

\section{Patient consent for publication}

Not applicable.

\section{Competing interests}

The authors declare that they have no competing interests.

\section{References}

1. Global Burden of Disease Cancer Collaboration, Fitzmaurice C, Abate D, Abbasi N, Abbastabar H, Abd-Allah F, Abdel-Rahman O, Abdelalim A, Abdoli A, Abdollahpour I, et al: Global, regional, and national cancer incidence, mortality, years of life lost, years lived with disability, and disability-adjusted life-years for 29 cancer groups, 1990 to 2017: A systematic analysis for the global burden of disease study. JAMA Oncol 5: 1749-1768, 2019 (Online ahead of print).

2. Chen W, Zheng R, Baade PD, Zhang S, Zeng H, Bray F, Jemal A, Yu XQ and He J: Cancer statistics in China, 2015. CA Cancer J Clin 66: 115-132, 2016.

3. Song Z, Wu Y, Yang J, Yang D and Fang X: Progress in the treatment of advanced gastric cancer. Tumour Biol 39: 1010428317714626 , 2017.

4. Digklia A and Wagner AD: Advanced gastric cancer: Current treatment landscape and future perspectives. World $\mathrm{J}$ Gastroenterol 22: 2403-2414, 2016.

5. Shearer RF, Iconomou M, Watts CK and Saunders DN: Functional roles of the E3 ubiquitin ligase UBR5 in cancer. Mol Cancer Res 13: 1523-1532, 2015.

6. Li W, Bengtson MH, Ulbrich A, Matsuda A, Reddy VA, Orth A, Chanda SK, Batalov S and Joazeiro CA: Genome-wide and functional annotation of human E3 ubiquitin ligases identifies MULAN, a mitochondrial E3 that regulates the organelle's dynamics and signaling. PLoS One 3: e1487, 2008. 
7. Callaghan MJ, Russell AJ, Woollatt E, Sutherland GR, Sutherland RL and Watts CK: Identification of a human HECT family protein with homology to the Drosophila tumor suppressor gene hyperplastic discs. Oncogene 17: 3479-3491, 1998.

8. Henderson MJ, Russell AJ, Hird S, Muñoz M, Clancy JL, Lehrbach GM, Calanni ST, Jans DA, Sutherland RL and Watts CK: EDD, the human hyperplastic discs protein, has a role in progesterone receptor coactivation and potential involvement in DNA damage response. J Biol Chem 277: 26468-26478, 2002.

9. Matsuura K, Huang NJ, Cocce K, Zhang L and Kornbluth S: Downregulation of the proapoptotic protein MOAP-1 by the UBR5 ubiquitin ligase and its role in ovarian cancer resistance to cisplatin. Oncogene 36: 1698-1706, 2017.

10. Scialpi F, Mellis D and Ditzel M: EDD, a ubiquitin-protein ligase of the N-end rule pathway, associates with spindle assembly checkpoint components and regulates the mitotic response to nocodazole. J Biol Chem 290: 12585-12594, 2015.

11. Cojocaru M, Bouchard A, Cloutier P, Cooper JJ, Varzavand K, Price DH and Coulombe B: Transcription factor IIS cooperates with the E3 ligase UBR5 to ubiquitinate the CDK9 subunit of the positive transcription elongation factor B. J Biol Chem 286 5012-5022, 2011.

12. Munoz MA, Saunders DN, Henderson MJ, Clancy JL, Russell AJ, Lehrbach G, Musgrove EA, Watts CK and Sutherland RL: The E3 ubiquitin ligase EDD regulates S-phase and G(2)/M DNA damage checkpoints. Cell Cycle 6: 3070-3077, 2007.

13. McDonald WJ, Sangster SM, Moffat LD, Henderson MJ and Too CK: alpha4 phosphoprotein interacts with EDD E3 ubiquitin ligase and poly(A)-binding protein. J Cell Biochem 110: $1123-1129,2010$

14. Zhang Z, Zheng X, Li J, Duan J, Cui L, Yang L, Zhang L, Zhang Q and Wang X: Overexpression of UBR5 promotes tumor growth in gallbladder cancer via PTEN/PI3K/Akt signal pathway. J Cell Biochem, Feb 18, 2019 (Online ahead of print)

15. Meissner B, Kridel R, Lim RS, Rogic S, Tse K, Scott DW, Moore R, Mungall AJ, Marra AM, Connors JM, et al: The E3 ubiquitin ligase UBR 5 is recurrently mutated in mantle cell lymphoma. Blood 121: 3161-3164, 2013.

16. Clancy JL, Henderson MJ, Russell AJ, Anderson DW, Bova RJ, Campbell IG, Choong DY, Macdonald GA, Mann GJ, Nolan T, et al: EDD, the human orthologue of the hyperplastic discs tumour suppressor gene, is amplified and overexpressed in cancer. Oncogene 22: 5070-5081, 2003.

17. O'Brien PM, Davies MJ, Scurry JP, Smith AN, Barton CA, Henderson MJ, Saunders DN, Gloss BS, Patterson KI, Clancy JL, et al: The E3 ubiquitin ligase EDD is an adverse prognostic factor for serous epithelial ovarian cancer and modulates cisplatin resistance in vitro. Br J Cancer 98: 1085-1093, 2008.

18. Bradley A,Zheng H,Ziebarth A, Sakati W, Branham-O'Connor M, Blumer JB, Liu Y, Kistner-Griffin E, Rodriguez-Aguayo C, Lopez-Berestein G, et al: EDD enhances cell survival and cisplatin resistance and is a therapeutic target for epithelial ovarian cancer. Carcinogenesis 35: 1100-1109, 2014.
19. Eblen ST and Bradley A: MOAP-1, UBR5 and cisplatin resistance in ovarian cancer. Transl Cancer Res 6 (Suppl 1): S18-S21, 2017.

20. Yang M, Jiang N, Cao QW, Ma MQ and Sun Q: The E3 ligase UBR5 regulates gastric cancer cell growth by destabilizing the tumor suppressor GKN1. Biochem Biophys Res Commun 478: 1624-1629, 2016.

21. Akagi T, Yoshino T, Motoi M, Takata H, Yano S, Miyoshi I, Oka T and Ohtsuki Y: Isolation of virus-producing transformants from human gastric cancer cell line, HGC-27, infected with human T-cell leukemia virus type I. Jpn J Cancer Res 79: 836-842, 1988.

22. Akagi T and Kimoto T: Human cell line (HGC-27) derived from the metastatic lymph node of gastric cancer. Acta Med Okayama 30: 215-219, 1976.

23. Ding X, Zhu J, Wang X, Zhou W, Wu K, Zhou Z, Zhou K, Wu D, Jiao J, Xia Y and Wang X: Different cytotoxicity of disinfection by-product haloacetamides on two exposure pathway-related cell lines: Human gastric epithelial cell line GES-1 and immortalized human keratinocyte cell line HaCaT. Sci Total Environ 692: 1267-1275, 2019

24. Ke Y, Ning T and Wang B: Establishment and characterization of a SV40 transformed human fetal gastric epithelial cell line-GES-1. Zhonghua Zhong Liu Za Zhi 16: 7-10, 1994 (In Chinese).

25. Livak KJ and Schmittgen TD: Analysis of relative gene expression data using real-time quantitative PCR and the 2(-Delta Delta C(T)) method. Methods 25: 402-408, 2001.

26. Agnes A, Biondi A, Laurino A, Persiani R and D'Ugo D: Global updates in the treatment of gastric cancer: A systematic review. Part 1: Staging, classification and surgical treatment. Updates Surg 72: 341-353, 2020.

27. Socovich AM and Naba A: The cancer matrisome: From comprehensive characterization to biomarker discovery. Semin Cell Dev Biol 89: 157-166, 2019.

28. Flack JE, Mieszczanek J, Novcic N and Bienz M: Wnt-dependent inactivation of the groucho/TLE co-repressor by the HECT E3 ubiquitin ligase Hyd/UBR5. Mol Cell 67: 181-193.e5, 2017.

29. Li CG, Mahon C, Sweeney NM, Verschueren E, Kantamani V, Li D, Hennigs JK, Marciano DP, Diebold I, Abu-Halawa O, et al: PPAR $\gamma$ interaction with UBR5/ATMIN promotes DNA repair to maintain endothelial homeostasis. Cell Rep 26: 1333-1343.e7, 2019.

30. Lee YR, Chen M and Pandolfi PP: The functions and regulation of the PTEN tumour suppressor: New modes and prospects. Nat Rev Mol Cell Biol 19: 547-562, 2018.

This work is licensed under a Creative Commons Attribution-NonCommercial-NoDerivatives 4.0 International (CC BY-NC-ND 4.0) License. 